FORMATION Formation emploi

Revue française de sciences sociales

115 | Juillet-Septembre 2011

Le développement de l'aide à la personne : quelle professionnalisation?

\title{
Le rôle ambivalent des associations d'aide à domicile dans la professionnalisation des emplois et des salariées
}

The ambivalent role of home care associations in jobs and employees

professionalization

Die doppelwertige Rolle der der Vereine für Haushaltshilfe bei der

Professionalisierung der Arbeitsplätze und der Beschäftigten

El papel ambivalente de las asociaciones de ayuda a domicilio en la

profesionalización de los empleos y las asalariadas

\section{Emmanuelle Puissant}

\section{OpenEdition}

Journals

Édition électronique

URL : http://journals.openedition.org/formationemploi/3431

DOI : 10.4000/formationemploi.3431

ISSN : 2107-0946

Éditeur

La Documentation française

Édition imprimée

Date de publication : 23 septembre 2011

Pagination : $37-50$

ISSN : 0759-6340

Référence électronique

Emmanuelle Puissant, « Le rôle ambivalent des associations d'aide à domicile dans la

professionnalisation des emplois et des salariées », Formation emploi [En ligne], 115 | Juillet-Septembre 2011, mis en ligne le 24 septembre 2013, consulté le 30 octobre 2020. URL : http://

journals.openedition.org/formationemploi/3431; DOI : https://doi.org/10.4000/formationemploi.3431 


\section{DOSSIER}

\section{Le rôle ambivalent des associations d'aide à domicile dans la professionnalisation des emplois et des salariées'}

Emmanuelle Puissant ${ }^{\star}$

La professionnalisation massive des emplois d'aide à domicile nécessite une rupture de l'interaction exclusive entre aides à domicile et particuliers, et le développement de dispositifs d'intermédiation, notamment grâce au déploiement de l'encadrement intermédiaire.

Les activités d'aide à domicile auprès des personnes âgées, handicapées, et des familles en difficultés étaient, avant les années 40, réalisées de manière informelle par les sœurs, filles, belles-filles, voisines qui aidaient les personnes de la famille non autonomes. Au lendemain de la seconde guerre mondiale, ces activités ont été organisées dans le cadre d'associations, principalement issues des mouvements catholiques et ouvriers. Issu de la sphère domestique informelle, le secteur de l'aide à domicile a été investi

\footnotetext{
${ }^{1}$ Le présent article est issu d'une contribution pour le colloque de l'Association d'Économie Sociale, qui a eu lieu en septembre 2010, à Charleroi (Belgique). Il est le fruit d'une enquête de terrain réalisée dans le cadre du travail de thèse (voir encadré méthodologique). Il propose une mise en dialogue des résultats des différents travaux de terrain mentionnés. Il se situe, d'un point de vue disciplinaire, au carrefour de trois types d'approches : la socioéconomie des services, la socio-économie du travail, et l'économie sociale et solidaire.
}

\footnotetext{
* Emmanuelle Puissant est économiste, docteure en sciences économiques, diplômée de I'université de Grenoble, actuellement directrice scientifique à l'ADEES Rhône-Alpes (Association pour le développement des études économiques et sociales). Domaines d'investigation : économie sociale et solidaire, socio-économie du travail, socio-économie des services, travail et emploi des femmes. Elle a publié notamment : Lamotte B., Puissant E., "Segmentation du marché du travail et précarité de l'aide à domicile. Comment les partenaires sociaux peuvent-ils agir? », in Économies et Sociétés, Série "Socio-économie du travail ", $A B n^{\circ} 32,7 / 2010$. Artis A., Demoustier D., Puissant $\mathrm{E}$., "Le rôle de l'économie sociale et solidaire sur les territoires, six études de cas comparées ", Revue des Études Coopératives, Mutualistes et Associatives (Recma), n 314 (2009).
} 
par les politiques sociales (politiques familiales, politiques gérontologiques) et par les politiques de l'emploi (développement de contrats aidés, investissement public dans ce secteur considéré comme un « gisement potentiel d'emplois »).

Dans ce contexte, les organisations du secteur ont engagé, depuis les années 70, un processus de professionnalisation, au sens de la constitution d'une profession, telle que définie par Aballea (2005) : «Une activité ayant un objet spécifique », reposant sur une «qualification » (c'est-à-dire « un savoir constitué et validé »), mise en œuvre selon une « déontologie » (c'est-à-dire « des règles normatives reconnues et publicisées »), «dotant ceux qui la pratiquent d'un titre et d'un statut distinctifs » (p. 55). Lorsque les activités existent déjà, la constitution d'une profession implique la reconnaissance et la clarification des activités réalisées.

Ces processus de professionnalisation se sont nettement accélérés dans les années 2000, avec la loi rénovant l'action sociale et médico-sociale du 2 janvier 2002, dite « loi 2002-2», ainsi que l'accord de branche du 29 mars 2002 sur les rémunérations et les qualifications des salariés de la branche professionnelle « aide à domicile », qui établit quatre niveaux d'aide à domicile selon le niveau de diplômes ${ }^{2}$. Or, dans ce secteur, l'histoire et l'actualité du fonctionnement associatif témoignent d'une ambivalence quant à la professionnalisation des emplois ${ }^{3}$ et des personnes ${ }^{4}$ intervenant au domicile des particuliers (ou « aides à domicile »). Le présent article se propose précisément de présenter cette ambivalence de l'acteur associatif, oscillant entre travail domestique et travail collectif. En effet, notre analyse révèle que les associations contribuent au maintien des salariées dans un rapport domestique à leur travail ; cependant, en tant qu'acteur collectif historique du secteur, elles sont aussi en mesure de réunir les conditions pour favoriser l'émergence d'une réelle profession, reconnue comme telle.

\footnotetext{
${ }^{2}$ Ces quatre catégories sont, de la moins diplômée à la plus diplômée : le niveau A d'agent à domicile, le niveau $\mathrm{B}$ d'employé à domicile, le niveau $\mathrm{C}$ d'auxiliaire de vie sociale, et le niveau $\mathrm{D}$ qui comprend une partie des encadrants intermédiaires, et surtout les techniciennes d'intervention sociale et familiale (TISF).

${ }^{3}$ Clarification des postes de travail et des responsabilités qui incombent à chaque niveau de la hiérarchie salariale.

${ }^{4}$ Reconnaissance des compétences des salariés (par la qualification) et amélioration de leurs compétences (par des formations).
}

\section{RAPPORT DOMESTIQUE AU TRAVAIL ET FREINS À LA RECONNAISSANCE DU TRAVAIL SALARIÉ}

La reconnaissance du travail salarié, au sens de l'analyse interactionniste des professions (Vasselin, 2002), nécessite un éloignement de la sphère domestique, c'est-à-dire une objectivation et une division des tâches effectuées (Hughes, 1952). Or, la sphère domestique reste très prégnante dans les associations, freinant alors la reconnaissance de l'emploi salarié.

Les difficultés de reconnaissance de l'emploi salarié s'expliquent d'abord par la prégnance d'un rapport domestique au travail, qui peut se comprendre par la proximité de l'emploi associatif avec le travail bénévole. De plus, le travail bénévole contribue à maintenir centrale la figure du destinataire des services, reléguant au second plan celle de la salariée ${ }^{5}$. Enfin, le contexte de fort émiettement du paysage associatif, dans ce secteur, et donc des employeurs, renforce ces difficultés d'affirmation et de reconnaissance de l'emploi salarié. Ces trois éléments nous apparaissent comme des freins à la professionnalisation. Ils favorisent en effet le maintien d'un rapport domestique au travail d'aide à domicile ; en outre, ils constituent des obstacles à la reconnaissance des compétences requises comme étant des compétences professionnelles.

\section{La proximité avec le travail bénévole}

Le premier frein à l'affirmation de la relation salariale concerne la proximité entre le travail bénévole et le travail salarié (Hely, 2009). Cette proximité est de deux ordres. La première est historique. Elle résulte du fait que le bénévolat des personnes peut être à l'origine de leur emploi salarié : "Les salariés qui n'ont pas été bénévoles dans l'association avant d'être recrutés dans le cadre de ce dispositif [« nouveaux emplois, nouveaux services »] conservent une probabilité plus forte $(30,2 \%$ de chances contre $24 \%)$ d'obtenir une rémunération mensuelle nette supérieure à 988 euros

\footnotetext{
5 Selon l'Enquête Emploi en continu pour l'année 2007, plus de $98 \%$ des salariés sont des femmes, c'est pourquoi nous employons le féminin.
} 


\section{Encadré 1 \\ Méthodologie}

Monographie de la plus importante association d'aide à domicile (plus de 1200 salariés) du département de l'Isère, sur la base d'une immersion de plusieurs mois dans une association mandataire et prestataire $\left({ }^{*}\right)$.

Cette monographie résulte de plusieurs types d'outils : des entretiens ouverts (entretiens exploratoires ouverts), des entretiens semi-directifs (auprès d'une pluralité d'acteurs de l'association), une étude de la littérature grise de l'association létude des procès verbaux sur plusieurs années consécutives des Conseils d'Administration, des Assemblées générales, des rencontres de la direction avec le Comité d'entreprise, le Comité d'Hygiène, Santé et Conditions de Travail, des réunions droits d'expression, etc.), la participation régulière à des réunions d'équipes (réunions entre la direction et les responsables de secteur, réunion droits d'expression, temps de formation, temps d'analyses de la pratique), des temps d'échanges informels avec les salariés de différents niveaux de l'association, et enfin, le suivi de trois aides à domicile sur leurs lieux de travail pendant trois journées (présence durant les temps d'intervention, discussion avec les usagers, discussions avec l'aide à domicile - surtout pendant les trajets entre deux interventions).

Réalisation d'entretiens semi-directifs.

119 entretiens semi-directifs ont été menés, dont une partie dans le cadre de la monographie mentionnée plus haut : 52 aides à domicile, 21 responsables de secteur, 7 employeurs (administrateurs), 4 directeurs, 3 directeurs de ressources humaines ou directeurs de services, 2 responsables formation, 6 usagers, 1 financeur (Conseil général), 3 assistants sociaux de Conseils généraux, 5 personnels administratifs, 1 représentant d'enseignes nationales, 1 juriste du travail, 2 conseillères prud'homales, 4 représentants de fédérations d'associations, 6 responsables syndicaux.

Tous les entretiens ont été réalisés en face à face, sans rémunération des professionnels.

Les thèmes abordés ont varié selon le type d'acteurs, mais ils ont reposé sur un même déroulement: 1) Récit de vie introductif, présentation de la personne interrogée et de son parcours ; 2) Description du poste actuel (raisons de l'orientation vers le poste actuel, missions, contenu de l'activité, ancienneté dans la fonction, qualifications et place dans la grille salariale établie par l'accord de branche du 29 mars 2002, perspectives de promotions, etc.) ; 3) Principales difficultés rencontrées dans leur quotidien professionnel; 4) Dernière partie de l'entretien « libre » selon les souhaits des interlocuteurs.

$\left(^{*}\right)$ : Dans une association ou un service mandataire, la salariée est employée par le particulier chez lequel elle intervient. Elle relève donc de la convention collective du particulier employeur ; celui-ci paie un service pour l'aider dans la gestion administrative de l'emploi (contrats, fiches de paie, congés, etc.). Dans une association ou un service prestataire, la salariée est employée par un employeur collectif (public ou privé lucratif ou non lucratif), ici associatif. Elle relève donc d'une des trois conventions collectives de l'emploi prestataire (convention collective de 1970 de l'aide familiale, celle de 1970 de l'aide à domicile en milieu rural, ou celle de 1982 de l'aide à domicile non lucrative), en passe d'être regroupées dans une convention collective unique (actuellement en cours de négociation).

que ceux qui l'ont été avant d'être embauchés » (Hely, op. cit. p. 90).

La seconde proximité est issue de la relation spécifique au secteur associatif entre les bénévoles (dirigeants et employeurs) et les salariés, relation de type familial. Globalement, et de manière plus exacerbée encore au sein des petites associations, les présidents sont souvent des figures locales. Cette position particulière sur le territoire est déterminante dans le choix d'être administrateur d'une association, et donc d'endosser la responsabilité d'employeur. L'envie d'employer et de diriger des salariés intervient peu dans le choix d'occuper une telle fonction. Ainsi, paradoxalement, si être administrateur est un choix, être employeur l'est moins souvent. Les employeurs ont ainsi rarement appris à l'être (aucune formation particulière 
en droit du travail notamment) ; or, les formations pour les bénévoles sont rares. De plus, l'utilisation abondante, par les employeurs associatifs, de contrats aidés, dérogatoires au Code du Travail, et l'absence d'interlocuteurs salariés (les emplois de coordination et d'encadrement intermédiaire, quand ils existent, peuvent être endossés par des bénévoles dans les petites associations) constituent autant d'éléments qui contribuent à « dénier aux travailleurs associatifs leur statut de salarié et, de ce fait, à favoriser le développement d'attitudes paternalistes dans le comportement des employeurs du monde associatif » (Hely, op. cit., p. 86).

\section{La centralité de la figure de l'usager}

Dans leur fonctionnement, les associations ont toujours accordé une place centrale à la relation avec l'usager. Cette centralité est aujourd'hui mise en question ; cependant, elle continue à marquer le secteur de l'aide à domicile et explique pourquoi la relation salariale a peiné à s'imposer.

L'histoire des mouvements charitables et religieux témoigne que les premières associations valorisaient largement le « don de soi » et que la professionnalisation n'était pas une priorité. Le travail d'aide à domicile était d'ailleurs, au départ, un travail bénévole, réalisé dans une logique d'aide charitable et d'entraide entre femmes d'ouvriers. Même lors des premières formes de rémunérations, ces emplois étaient moins considérés comme de vrais emplois que comme du bénévolat indemnisé (Puissant, 2007). L'étude des statuts des premières associations atteste que la préservation de l'autonomie et de la dignité de l'usager, grâce à sa participation directe aux services, constitue le premier objectif des associations dès leur création. Le souci de créer une « vraie » profession est l'objectif second.

Par exemple, les services de l'aide familiale populaire, créés en 1942 dans le cadre des jeunesses ouvrières chrétiennes, visent : «[...] tout d'abord à faire la promotion du monde ouvrier, à assurer la participation des usagers, à leur donner l'occasion d'une prise de responsabilité et à leur permettre ainsi de ne pas se sentir de perpétuels assistés » (Bonamy, 1984, p. 299). Ainsi, la promotion de la classe ouvrière, par le partage des responsabilités et par la participation des ouvriers (usagers) à la réalisation de services d'entraide, est un élément essentiel. Il contribue à expliquer la forte compatibilité de l'organisation de ces services avec le statut associatif. Ce statut permet la « double qualité », à savoir d' " associer et de participer à l'activité » (Vienney, 1994), organisée dans le cadre d'une fonction employeur collective, bénévole, et assumée par un conseil d'administration qui permet la participation directe des usagers à l'activité de l'association. C'est le sens d'une motion votée par l'assemblée générale du service des aides familiales au Congrès du mouvement familial populaire, en 1949 : «Pour que notre service ne s'aligne pas sur le type des services sociaux actuels, l'assemblée condamne toutes les associations qui ne seraient pas gérées avec les USAGERS ${ }^{6}$ eux-mêmes » (citée par Duriez, Nizey, 2002, p. 206). On peut ainsi parler de « relation d'usage » (Dussuet, Puissant, 2010), qui constitue toujours un point d'ancrage central pour les associations, permettant de rendre compte de la relation complexe entre salariés et usagers. Elle renvoie à une relation institutionnalisée d'adaptabilité conjointe : l'intervenant et l'usager définissent, à l'usage, l'activité réalisée (Bourquin, 2001).

La signature des premières conventions collectives, dans les années $70^{7}$, institue l'emploi salarié dans les services, au sens de la rétribution du travail par un salaire mais aussi d'une réglementation précise $\mathrm{du}$ travail (ce qui équivaut, en droit du travail, à un échange entre la protection du salarié contre sa subordination à l'employeur). L'affirmation progressive de l'association comme employeur, suite aux développements de politiques d'emploi (politiques d'insertion, création et développement des contrats aidés, etc.) dans les années 80 , accentue encore la tension naissante entre la relation salariale et la relation d'usage. Ainsi, en dehors de toute référence au travail bénévole, les associations ont commencé à recruter des aides à domicile directement sur le marché de l'emploi, à l'extérieur de leurs réseaux. D'une part, l'affirmation de l'aide à domicile salariée dans le domicile privé des usagers a déstabilisé la logique selon laquelle seule l'adaptation des bénévoles et militants aux usagers et

\footnotetext{
${ }^{6}$ En lettres capitales dans le texte.

${ }^{7}$ Convention collective nationale des organismes agréés de travailleuses familiales, signée le 2 mars 1970, et convention collective nationale des aides familiales rurales, signée le 3 mai 1970.
} 
la réponse à leurs besoins importaient. D'autre part, l'instauration d'un rapport de subordination entre salariées et militants a également contribué à éloigner l'aide à domicile de la relation d'usage (Dussuet, Loiseau, 2007). Ainsi, aujourd'hui, la double qualité a tendance à disparaître des statuts des associations ; les usagers apparaissant de plus en plus comme de simples bénéficiaires qui s'adressent à un prestataire de service. Le mode d'organisation associatif original qui reposait sur une participation des usagers en amont de la réalisation du service, permettant ainsi une co-conception du service, est donc remis en cause.

\section{L'émiettement associatif}

Enfin, le troisième frein à la reconnaissance du travail salarié est le morcellement des employeurs associatifs dans ce secteur : 48,9 \% des entreprises ${ }^{8}$ pour lesquelles travaillent les aides à domicile compteraient moins de cinquante salariés selon l'Enquête Emploi en continu 2007. Il existe des fédérations d'associations, comme l'Unadmr (Union nationale des associations d'aide à domicile en milieu rural) qui fonctionnent quasi-exclusivement sur la base d'associations locales de petite, voire de très petite taille $^{9}$. Les petites entreprises du secteur, associatives ou lucratives, sont enclines au développement de relations interpersonnelles fortes, davantage caractéristiques de la sphère domestique, que de relations professionnelles décrites par la sociologie des relations professionnelles (Reynaud, 1995).

C'est ainsi que les tensions, dans un cadre interindividuel, apparaissent plus comme des tensions entre des personnes que des tensions portant sur la relation de travail, et encore moins comme des conflits du travail. L'étude des conflits du travail dans les associations d'aide à domicile, qui ont abouti à la saisine du Conseil des Prud'hommes, en Isère, entre 2003 et 2006 (Puissant, 2006), montre que si les tensions dans la relation de travail sont globalement croissantes, quelle que soit la taille des associations, elles se manifestent

\footnotetext{
${ }^{8}$ Ce chiffre est toutefois à manier avec précaution, en raison de la très forte proportion d'entreprises non identifiées par le répertoire SIRENE (Système Informatique pour le Répertoire des Entreprises et de leurs Établissements) et de taille non renseignée : cette part s'élève à $31,2 \%$.

${ }^{9}$ La petite taille de ces entreprises ne permet notamment pas la mise en place d'instances représentatives du personnel.
}

par des conflits judiciarisés et affirmés essentiellement dans les associations de grande taille. Au sein des petites associations, ces tensions sont en général gérées de manière informelle, par les coordinateurs des services ou les présidents d'associations, les deux étant en général bénévoles. Ils aboutissent rarement à une saisine du Conseil des Prud'hommes ; ils se gèrent en interne : il s'agit de conflits plutôt interpersonnels, dans le sens où ils opposent deux personnes. L'extrait d'entretien ci-dessous montre que si dans les faits, les difficultés rencontrées par cette aide à domicile concernent le travail concret, ces difficultés sont vécues comme un conflit entre deux personnes ayant des caractères et des manières de travailler différents :

" J'interviens chez la même personne que X depuis quelques mois. Et bien c'est l'horreur. Sous prétexte qu'elle a eu le diplôme [le diplôme d'État d'auxiliaire de vie sociale], elle me laisse le linge sale à laver, la vaisselle à faire, et elle, elle se garde tout le côté relationnel du boulot. Alors que moi, je vais depuis plus longtemps qu'elle chez Madame Y. »

"Vous en avez parlé à la responsable de secteur, ou à des collègues?»

"Non, pas besoin. Qu'est-ce que vous voulez qu'elle fasse? C'est un problème entre X et moi. Un jour, je vais la prendre entre quatre yeux et lui dire d'arrêter de faire ça » (Entretien avec une aide à domicile, catégorie A, sans qualification, travaillant dans une petite association rurale de l'agglomération grenobloise).

Le même type de dysfonctionnement existe dans une association grenobloise comptant plus de 1000 salariés. Dans ce cas, le problème a été discuté lors de réunion «droits d'expression » entre collègues; les déléguées syndicales ont été alertées, les responsables de secteur prévenues et impliquées. Ce dysfonctionnement est alors vécu comme un problème d'organisation du travail, qu'il faut régler collectivement.

De surcroît, les petites associations locales ont souvent été créées par des personnalités charismatiques. Leur gestion peut, dans un contexte caractérisé par des relations interpersonnelles fortes, donner lieu à une forme de paternalisme ; cela freine encore davantage l'affirmation de la relation salariale dans sa globalité, avec ce qu'elle implique en termes de négociations collectives, de gestion des carrières professionnelles, 
de formations, de qualifications, etc. Ainsi, les entretiens réalisés témoignent de différences importantes dans le rapport au travail des aides à domicile, selon qu'elles travaillent au sein d'importantes associations urbaines, ou dans de petites associations rurales. Dans le second cas, le discours des coordinateurs ou présidents d'associations bénévoles et celui des aides à domicile sont très proches : les thèmes du salaire, de la qualification (singulièrement de l'obtention de diplômes par la VAE - validation des acquis de l'expérience) sont peu souvent abordés de manière spontanée. Les thèmes prédominants portent davantage sur les relations affectives avec les usagers. À l'inverse, dans les associations de plus grande taille, plutôt urbaines dans notre échantillon, les salariées en viennent quasi systématiquement à parler spontanément du salaire et des conditions de travail, d'emploi et de qualification. On peut supposer que cette forme de paternalisme est plus prononcée encore lorsqu'il s'agit de petites associations non affiliées à une fédération. Les fédérations peuvent en effet constituer un facteur de contrôle (d'application du Code du travail notamment) et de régulation (en cas d'importants conflits internes, en cas de difficultés financières, etc.). Ce paternalisme peut être encore exacerbé par l'absence de cadres intermédiaires salariés entre les bénévoles employeurs (présidents et administrateurs ${ }^{10}$ ) et les intervenants à domicile, comme c'est souvent le cas dans les petites structures.

Ainsi, le rappel de l'histoire des associations a mis en exergue les freins réels à la reconnaissance de l'emploi salarié, qui apparaît comme une condition à l'éloignement de la sphère domestique. Or, il nous semble justement que ces trois éléments participent du maintien d'un registre d'action domestique, au sens de Salais et Storper (1993). Un registre d'action est partagé par le producteur et le demandeur, qui se coordonnent sur la base de cet accord. Dans l'aide à domicile, le registre d'action partagé par les usagers, les aides à domicile et par certains employeurs, appartient au «monde de production interpersonnel», au sens où l'identité des objets (ou services) produits est inséparable des personnes qui le produisent et l'utilisent. Ce monde de production, très proche $\mathrm{du}$ « monde de justification domestique » (Boltanski,

${ }^{10}$ Les « administrateurs » sont les membres du conseil d'administration, qui est l'employeur légal des aides à domicile.
Thevenot, 1991), est fondé sur des relations interpersonnelles fortes, l'instauration d'une confiance et d'une proximité, sur le modèle des relations tissées entre les membres d'un même foyer. C'est ainsi qu'à partir des travaux d'Aballea sur les assistantes maternelles, on peut parler d'un statut quo parmi les acteurs de l'aide à domicile autour du caractère féminin et non professionnel des carrières dans ce secteur : il s'agit d'emplois dits féminins au double sens qu'ils sont occupés à hauteur de 98 \% par des femmes, et qu'ils apparaissent comme "naturellement féminins », prolongeant le travail que les femmes réalisent dans la sphère domestique (Dussuet, 2005 ; Avril, 2007). Les aides à domicile chercheraient à prolonger leur expérience domestique dans la sphère professionnelle; et ce, non pour faire carrière au sens d'une montée en qualification et/ou en responsabilités, mais pour être sur le marché du travail en usant de compétences déjà acquises dans la sphère domestique.

Ce statut quo est donc issu du registre d'action domestique, dans lequel les relations sont interpersonnelles, affectives, floues et informelles. Cette prégnance du registre domestique dans les activités d'aide à domicile est certes un facteur de l'intérêt des salariés pour leur travail ; cependant, elle constitue aussi un frein à la reconnaissance des aides à domicile comme vraies professionnelles, détentrices d'un savoir-faire particulier et validé. On peut atténuer ou du moins circonscrire ce registre domestique par l'organisation collective des services d'aide à domicile. Ainsi, l'employeur collectif historique que représente l'association nous paraît être le lieu de l'éloignement du registre domestique, ou du moins de la précision et réduction de la part d'informel, inhérente à ce type d'activités. La structuration, la formalisation et l'organisation collectives apparaissent comme des enjeux forts, en vue de la reconnaissance de ces activités comme activités professionnelles.

\section{LA RE-CRÉATION D'UN TRAVAIL COLLECTIF COMME LEVIER DE PROFESSIONNALISATION}

La situation française se caractérise par la prépondérance de l'emploi direct. En France, il concerne, 
en 2006, $74 \%$ des heures effectuées au domicile des particuliers, et $80 \%$ des salariés travaillant au domicile des particuliers (Chol, 2008). Une convention collective existe depuis 1999. Toutefois, elle est bien en deçà des conventions régissant le travail dans le cadre de structures prestataires, notamment en matière de prise en compte des temps de trajets entre deux domiciles, considérables dans ces métiers de la mobilité, et de droit à la formation (Jany-Catrice, 2009). Or, l'emploi direct est caractérisé par de faibles garanties statutaires, mais aussi par un travail morcelé (horaires atypiques et éclatés, multiplicité des lieux de travail, etc.) et isolé (absence d'un lieu collectif de travail, absence de collègues de travail, travail en interaction exclusive avec les destinataires des services), proche du travail domestique, qui éloigne les salariés des possibilités de suivi de formations ou d'obtention de qualifications.

Face à cette situation, des fédérations d'associations militent pour la reconnaissance des emplois et des qualifications dans les associations prestataires du secteur, c'est-à-dire des associations employeuses, qui engagent les intervenants à domicile sous statut de salariés. Des fédérations historiques chargées de défendre les familles populaires militent, d'une part, pour la reconnaissance des emplois "féminins 》 comme des emplois requérant des compétences professionnelles particulières; d'autre part, pour que les interventions à domicile soient reconnues comme nécessitant des formations et des diplômes, tel celui de technicienne d'intervention sociale et familiale (anciennes travailleuses familiales) ${ }^{11}$. La collectivisation du travail des aides à domicile, telle qu'elle est permise par la structuration associative, est une condition de la reconnaissance de ces activités professionnelles. La re-création d'un travail collectif, dans ce contexte d'un travail par nature éclaté et morcelé, passe, dans les associations, par l'élaboration d'un cadre de travail explicite et collectif, par la constitution d'espaces collectifs de travail, tels qu'ils peuvent être incarnés par les postes de coordination et d'encadrement intermédiaire ; ces deux éléments contribuant à créer un cercle vertueux de la professionnalisation.

\footnotetext{
${ }^{11}$ C'est le cas de la Fédération nationale des associations de l'Aide familiale populaire de la Confédération syndicale des familles Fnaapf-Csf - (pour l'histoire de la professionnalisation des travailleuses familiales, voir Dussuet, Loiseau, op. cit.).
}

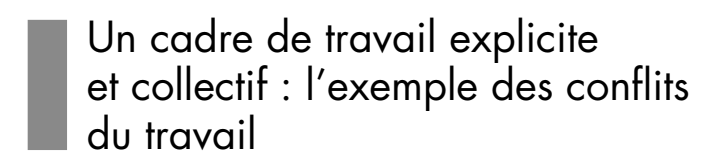

Les associations prestataires proposent aux salariés un cadre de travail davantage explicite et collectif que l'emploi direct. L'explicitation du cadre de travail, la formalisation des règles et des procédures sont des conditions à l'élaboration d'un «système de relations professionnelles » au sein d'un collectif de travail, dans le sens de relations portant sur les salaires, l'emploi, la qualification, la carrière professionnelle, et plus globalement l'organisation (Reynaud, op . cit., p. 3). La formalisation des règles permet de clarifier les droits et devoirs des différentes parties. Elle prévoit des recours possibles en cas de non respect des règles établies. C'est ainsi que les conflits du travail aboutissant à la saisine du Conseil des Prud'hommes se développent considérablement dans les associations.

La notion de conflits du travail avait peu de sens lors de l'émergence des premières associations du secteur; et ce en raison, d'une part, de l'absence d'un rapport juridique de subordination régi par le Code de travail dans le cadre du travail bénévole ; d'autre part, du manque d'identification claire des employeurs et des salariés dans le cadre du travail salarié. Les associations dites «paternalistes » ont souvent réglé les conflits émergents, essentiellement interindividuels, de manière domestique. Le conflit est alors défini comme pathologique, exprimant une opposition de personnes pouvant aggraver un dysfonctionnement de l'organisation. La gestion domestique des différends suppose l'existence d'une loi d'entreprise interne qui prévaut sur les règles de droit. L'émergence d'un conflit est alors difficile au regard du déséquilibre important dans le rapport de force entre employeur et employé. Leplay (2005) identifie la perte de confiance comme étant le principal facteur de conflits dans le cadre d'une gestion domestique du travail :

" Avant, moi, je ne comptais pas mes heures, c'est clair. Quand ma responsable me demandait, au pied levé, de venir chez une personne âgée, je le faisais sans problème. On avait le même intérêt, ma responsable et moi, le bien-être de la personne âgée. Mais maintenant, on ne m'y reprendra plus. On a l'impression que maintenant, la direction se moque des bénéfciaires, et que la seule chose qui les intéresse, c'est de 
faire des économies. » (Entretien avec une auxiliaire de vie sociale - obtention du Diplôme d'Auxiliaire de Vie Sociale - DEAVS - par la validation des acquis de l'expérience), déléguée du personnel, travaillant pour la même association depuis 13 ans.

L'engagement bénévole pour une cause « juste » pouvait suffire à légitimer un investissement important ; à présent, la rétribution reconnaît des compétences aux intervenantes à domicile, leur travail s'organise et la demande des usagers devient plus exigeante. Dans ce contexte émergent des « relations de travail » qui révèlent parfois des problèmes d'entente, de coordination ou de communication, susceptibles de mettre en difficulté la communauté de travail qui se construit :

"Dès lors que l'on travaille avec d'autres et que la réussite de notre action dépend d'autres personnes, il y a forcément des conflits d'intérêts, de représentation, ou tout simplement de personnes. C'est normal, et il ne faut même pas essayer de l'empêcher. C'est la "vie en société". Seulement, pendant des années, dans les associations, on ne le savait pas! On pensait, de l'extérieur, que dans une association tout le monde s'entendait bien, parce que tout le monde adhérait au même projet et travaillait là par choix, et donc qu'il ne pouvait pas y avoir de relations professionnelles vraiment conflictuelles... » (Entretien avec une conseillère prud'homale, côté employeur, du Conseil des Prud'hommes de Montbrison ; cette personne a aussi été pendant longtemps directrice d'une association d'aide à domicile).

Avec le développement des activités et la croissance des associations, de réels « conflits du travail» ont émergé. La formalisation et la clarification des règles et procédures ont induit une judiciarisation croissante des conflits du travail. La recrudescence des recours au Conseil des Prud'hommes, depuis les années 2000, renseigne ainsi davantage sur l'évolution de l'expression des conflits que sur leur augmentation réelle. Elle est une conséquence de la structuration des relations professionnelles, elle en est aussi un vecteur : ce tribunal permet à chaque acteur d'apprendre son rôle respectif, et de comprendre les outils juridiques existants de régulation du travail et des relations professionnelles. Ainsi, « la confrontation judiciaire n'épuise pas les conflits » (Damesin, Pelisse, 2004).
Par ailleurs, l'éclatement de conflits du travail devant le Conseil des Prud'hommes témoigne de la collectivisation du travail dans les associations d'aide à domicile.

\section{Un espace collectif de travail : l'exemple de l'encadrement intermédiaire}

Dans un contexte d'emplois éclatés et isolés, les associations réunissent les conditions pour permettre la constitution d'un espace collectif de travail. Cet espace est constitué d'un lieu de rencontre (au siège social pour les petites associations, dans les antennes de secteurs pour les plus grosses). Ce lieu favorise l'instauration de relations entre collègues (échanges sur les pratiques, les plannings, les usagers communs), des temps collectifs (réunions droit d'expression, restitutions collectives des fiches de paie, réunions avec les responsables de secteur, temps d'analyse de la pratique avec des psychologues), des instances de représentation du personnel, et parfois des délégués syndicaux. Si la syndicalisation est globalement faible parmi les aides à domicile, elle est plus élevée chez les aides à domicile employées par une structure prestataire que chez les aides à domicile en emploi direct (Béroud S., Brugnot T., Garibay D., Vila D., 2009). La création d'un espace collectif de travail repose largement sur une spécificité du système prestataire : les emplois de coordination et d'encadrement intermédiaire, que nous nommerons, comme nombre d'associations, les « responsables de secteur ».

Les responsables de secteur, dont l'importance a déjà été pointée (Bouvier, Pelvillain, Santelma, 2010), jouent un rôle d'intermédiation essentiel entre les différentes parties prenantes du service. Ils contribuent en effet à collectiviser le travail des aides à domicile, qui résulte d'un système complexe de relations. Cette fonction-pivot rend donc cohérente la relation triangulaire de travail associatif à domicile.

$\mathrm{Au}$ cœur de cette relation triangulaire de travail, les responsables de secteur endossent un triple rôle d'intermédiation. D'abord, entre salariées et usagers : ils sont les seuls professionnels, autres que les aides à domicile, à se rendre aux domiciles des particuliers. Ils peuvent répondre aux besoins de médiation, par exemple pour redéfinir, avec l'usager, le champ de 
Schéma 1

Les emplois de coordination et d'encadrement intermédiaire, au cœur des relations entre les trois protagonistes del'aide à domicile

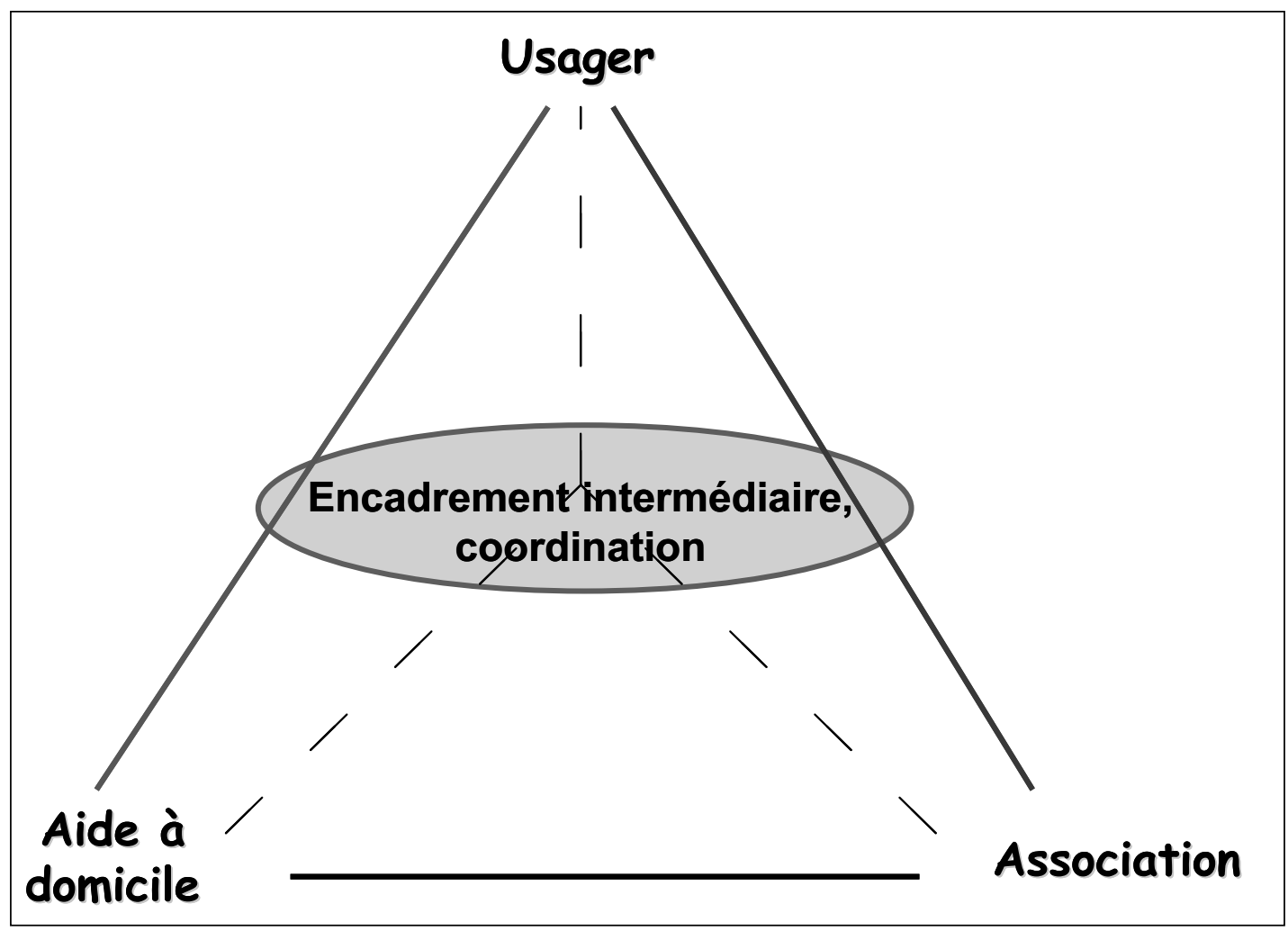

Source : Puissant Emmanuelle (2010).

compétence de l'aide à domicile. Lors des entretiens réalisés, les témoignages abondent pour confirmer l'importance de ce travail de coordination, d'écoute et de collectivisation pour les salariées :

« Selon que la responsable de secteur est bien ou pas, ça peut changer tout notre travail. Moi, franchement, je n'ai pas à me plaindre, je suis bien au courant de la situation quand je vais chez une personne, donc je ne me sens pas en danger. Mais des filles me disent que leur responsable ne leur fait pas confiance, ne les considère pas comme de vraies professionnelles, et au nom du secret professionnel, ne leur dit rien. Il en va parfois de la sécurité des salariées. On n'intervient pas de la même manière selon les situations, les pathologies des personnes. Donc quand on est briefée par notre responsable avant les interventions, cela se passe forcément mieux » (Entretien avec une déléguée du personnel d'une mutuelle de la Drôme - principal employeur d'aides à domicile du département -, auxiliaire de vie sociale (obtention du diplôme par la validation des acquis de l'expérience), en poste depuis 9 ans.)

Le deuxième rôle d'intermédiation se situe entre les salariés et l'association (en tant qu'employeur). Les employeurs sont peu en lien avec les intervenantes à domicile. Les seuls responsables hiérarchiques avec lesquels elles sont en lien sont les responsables de secteur.

Le troisième rôle d'intermédiation se situe entre les usagers et l'association (en tant que prestataire de service). Quand l'usager est insatisfait, il prévient le responsable de secteur, qui fait remonter l'information à l'équipe de direction. Inversement, quand elle envoie des informations postales aux usagers, l'équipe de direction compte sur les responsables de 
secteur pour veiller à leur bonne compréhension par les usagers.

L'importance de cet espace collectif de travail a notamment été mise en exergue par une étude récente portant sur les risques et les dispositifs de prévention dans les associations d'aide à domicile (Dussuet, 2009). Cette étude insiste sur le rôle d'outils ou de dispositifs de collectivisation du travail relatif à la prévention des risques professionnels, qui se déclinent aussi bien en termes de «pénibilités physiques» (déplacements, horaires atypiques, environnement de travail, fractionnement, intensification) qu'en termes de « risques psycho-sociaux 》 (travail émotionnel, face à face à huis clos, faible définition ou dévalorisation du travail). Face à ces deux formes de pénibilités ou risques professionnels, des outils de collectivisation du travail jouent le rôle de dispositifs potentiels de prévention. Ainsi, la définition de règles d'intervention, la description du travail, la gestion des plannings, les instances de médiation et de discussion autour des pratiques professionnelles, sont autant d'éléments en mesure de transformer l'environnement de travail, les conditions dans lesquelles il s'exerce, ainsi que le ressenti sur son travail.

\section{Un cercle vertueux de la professionnalisation?}

Nos entretiens témoignent de l'existence d'un cercle vertueux de la professionnalisation au sein des associations ; en effet, le travail collectif permettrait une distance par rapport au statut quo de la non-professionnalité. Les aides à domicile entreraient souvent dans ce secteur d'activité sans formations particulières, car elles « savent déjà faire » :

«Moi, je me suis beaucoup occupée de ma belle mère, elle était très malade, ce n'était vraiment pas facile. J'ai essayé de faire de mon mieux, et je pense qu'elle a réussi à être heureuse jusqu'à la fin. Alors, quand j'ai eu besoin de travailler, aide à domicile, ça s'est imposé tout seul. De toutes façons, entre vous et moi, je ne vois pas bien ce que j'aurais pu faire d'autre » (Entretien avec une agent à domicile - aide à domicile non qualifiée -, en emploi depuis 4 ans dans une association iséroise d'aide à domicile aux personnes âgées).
Plusieurs témoignages recueillis auprès de responsables de secteur confirment cette idée :

"Quand une aide à domicile est recrutée, ce n'est pas évident au début. Elles sont souvent méfiantes quand on les rencontre, elles ne comprennent pas qui on est, elles pensent qu'on est là pour les contrôler, pour les surveiller. Au début, le problème c'est qu'elles n'ont pas l'impression de travailler pour une association, dans une équipe. La seule chose qui leur importe, ce sont les familles, de faire correctement leur travail chez les familles, et le reste, c'est comme si c'était du travail en plus inutile. » (Entretien avec une responsable de secteur d'une association iséroise d'aide à domicile aux familles, titulaire du Caferuis, travaillant dans l'association depuis 12 ans)

Une fois la barrière de la première formation passée, le rapport au travail, et corrélativement à l'association (et aux collègues), changerait. Par les formations collectives, les aides à domicile commencent à se sentir appartenir à une équipe :

« Ce qui est marrant, c'est qu'à chaque fois, on se trouve face au même scénario : on se bagarre pour qu'elles viennent aux premières formations, et après, c'est comme si elles y prenaient goût, comme si du coup elles se rendaient compte de tout ce qu'elles ne savent pas, de tout ce qu'elles ont besoin d'apprendre pour être bien dans leur métier. Pas plus tard que la semaine dernière, une qui était vraiment récalcitrante au départ, est venue me demander des séances supplémentaires avec un psychologue. ») (Entretien avec une responsable de secteur, références citées précédemment)

Le même type de processus avec les réunions d'équipe (formelles ou informelles ${ }^{12}$ ) est évoqué : si les nouvelles recrues sont souvent sceptiques sur l'intérêt de rencontres régulières pour échanger sur les pratiques et les difficultés, elles en deviennent peu à peu demandeuses :

${ }^{12}$ Nous retenons ici l'existence de deux types de " réunions d'équipe » : les réunions formelles (comme les droits d'expression ou des réunions, ponctuelles ou régulières, entre les responsables de secteur et les aides à domicile de leur secteur), et les rencontres informelles (essentiellement le moment, à la fin de chaque mois, où la fiche de paye est remise aux aides à domicile par leur responsable de secteur). 
"Quand je suis rentrée dans ce métier, je ne savais pas vraiment ce que c'était. Je pensais seulement que j'allais m'occuper des personnes âgées. Je ne me rendais pas compte que je m'y prenais mal, que je ne m'économisais pas, que je pouvais moins me baisser pour moins me faire mal au dos, qu'il fallait que je fasse attention aux produits que j'utilisais. Je faisais tout ça machinalement, sans me rendre compte qu'en fait, ce métier comme d'autres, il s'apprend. » (Entretien avec une employée à domicile d'une association iséroise d'aide à domicile aux personnes âgées, engagée dans une démarche de validation des acquis de l'expérience, travaillant dans l'association depuis 13 ans)

Ce cercle vertueux peut aussi concerner l'investissement dans les instances représentatives du personnel dans les associations. Cette progression est bien résumée par une responsable de secteur d'une association d'aide à domicile aux familles, précédemment citée :

«Ce n'est pas rare de constater une progression bien nette : les personnes arrivent, pas qualifiées et réticentes à tout ce qui concerne le fonctionnement de l'association. Et puis elles font une formation, puis une deuxième. Puis elles nouent des liens réguliers avec les autres salariés du secteur. Et quand elles passent le stade de la qualification, là, elles commencent à s'intéresser aux déléguées du personnel, voire même se lancent là-dedans. Et nous, les responsables de secteur, on a un vrai rôle dans cette prise de conscience : on doit leur montrer qu'elles appartiennent à une équipe, à une association. » (Responsable de secteur d'une association iséroise d'aide à domicile aux familles, travaillant dans l'association depuis 12 ans)

Les associations, en tant qu'employeurs collectifs, semblent donc en mesure de mettre en œuvre une dynamique de professionnalisation.

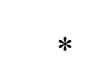

Ainsi, le fait que les aides à domicile elles-mêmes confirment l'idée qu'il s'agit d'un métier féminin, requérant des compétences « féminines », s'explique surtout par le maintien du registre d'action domestique dans ce secteur d'activité. Ce registre est accentué dans le cadre d'une interaction exclusive entre les deux parties prenantes du service. A contrario, il est en mesure d'être amoindri dans le cadre d'une organisation collective du travail ; cette organisation est schématisée par le triangle relationnel, au sein duquel l'encadrement intermédiaire joue un rôle crucial de coordination et de distanciation, comme en témoigne l'exemple associatif. Or, cette distanciation, au moins partielle, par rapport au registre domestique, est une condition à l'amorce et au développement d'un processus de professionnalisation des personnes et des emplois, au sens d'une reconnaissance de la spécificité et de la technicité des compétences requises pour exercer ce métier. L'intermédiation, telle qu'elle est mise en œuvre par les postes de coordination et d'encadrement intermédiaire, dans les associations, va bien au-delà de la mise en lien (par internet ou autres), l'échange de pratiques, la mise en place de lieux de rencontres, etc. Il s'agit d'une organisation collective du travail des aides à domicile, d'où l'importance de la schématisation triangulaire. En effet, on peut imaginer une relation en emploi direct (donc une relation duale et non triangulaire), qui n'est pas incompatible avec l'échange de pratiques entre collègues. Mais dans le triangle associatif, le point nodal du triangle (constitué par ces postes intermédiaires) change la nature du service rendu; en effet, il ne s'agit pas seulement d'un amoindrissement de l'isolement des aides à domicile, mais aussi d'une redéfinition des besoins des usagers (avec les usagers, et avec les salariées), d'une redéfinition du service rendu (avec un travail de délimitation de ce qui fait partie des devoirs des aides à domicile et de ce qui n'en fait pas partie, etc.). Selon que l'on se trouve face à une relation triangulaire ou duale, la nature même du service ne sera pas semblable.

En ce sens, la professionnalisation massive des emplois d'aide à domicile nécessite une rupture de l'interaction exclusive entre aides à domicile et particuliers, et le développement de dispositifs d'intermédiation. Ainsi l'offre de service est structurée en grande majorité par des organisations collectives ou au contraire par l'emploi direct caractérisé par une relation duale. Cela a des répercussions directes sur les possibilités effectives de la reconnaissance du secteur de l'aide à domicile comme secteur professionnel. Or, depuis les années 70 , les politiques publiques françaises encouragent l'emploi direct (exonérations successives de charges sociales et fiscales pour les particuliers 
employeurs, jusqu'à la création récente du chèque emploi service universel, dans le cadre de la loi 2005-32 de programmation pour la cohésion sociale, dite « Loi Borloo ») et le développement de la concurrence dans ce champ d'activités. Dans ce contexte, les financements publics sont restreints, et de plus en plus de conseils généraux remettent en cause la prise en charge, par la puissance publique, des coûts de fonctionnement des structures, comme les coûts des postes de coordination et d'encadrement intermédiaire. Ainsi, l'emploi direct est largement encouragé par les politiques publiques françaises, dans une stratégie de blanchiment du travail non déclaré d'une part, et de lutte contre le chômage, d'autre part ; dès lors, on peut s'interroger sur les possibilités effectives de ces professions d'être reconnues comme de réelles professions du secteur social, voire médico-social, requérant des compétences professionnelles à valoriser comme telles, notamment sur le plan salarial.
Plusieurs travaux (Jany-Catrice, 2008 ; Lefebvre, 2010) montrent l'inégalité des conditions de formation et de possibilités de qualifications entre l'emploi direct et l'emploi prestataire ; cependant, il apparaît nécessaire de mieux identifier le rôle professionnalisant ou non des différents prestataires : les associations sont-elles plus avancées que les entreprises lucratives en matière de reconnaissance et de valorisation des compétences et professions du secteur? Des travaux de comparaisons fines entre les conditions salariales et de travail à l'œuvre dans les associations, et dans les entreprises lucratives, sont nécessaires aujourd'hui. Il s'agit en effet de préciser les capacités effectivement professionnalisantes de l'employeur collectif historique du secteur ; acteur qui a participé à l'émergence des activités et à la structuration du secteur d'activité de l'aide à domicile, c'est à dire l'employeur associatif

\section{Bibliographie}

Aballea F. (2005), «La professionnalisation inachevée des assistantes maternelles », in Recherches et Prévisions, $\mathrm{n}^{\circ} 80$, juin.

Avril C. (2007), S'approprier son travail au bas $d u$ salariat. Les aides à domiciles pour personnes âgées, Paris, Thèse de doctorat.

Béroud S., Brugnot T., Garibay D., Vila D. (2009), Les syndicats face au phénomène des travailleurs pauvres en Europe, une approche compare, ONPES.

Boltanski L., Thevenot L. (1991), De la justification, les économies de la grandeur, Paris, Gallimard.

Bonamy B. (1984), « Pour un histoire des travailleuses familiales rurales et populaires », in Chauviere M., Dermenjian G., Guery L., Simonetti E., L'action familiale ouvrière et la politique de Vichy. Monde ouvrier et la presse des mouvements familiaux populaires. Être femme, être militante au mouvement populaire des familles, Les Cahiers du GRMF, $\mathrm{n}^{\circ} 3$, novembre.

Bourquin G. (2001), « Le travail social et la dimension de l'usage », in Humber C., Les usagers de l'action sociale : sujets, clients ou bénéficiaires ?, Paris, L'Harmattan.

Bouvier T., Pelvillain N., Santelmann P. (2010), «Services à la personne : renforcer l'encadrement intermédiaire pour accroître l'efficacité et l'attractivité des métiers ", in Formation Emploi ${ }^{\circ} 112$, octobre - décembre.

Chol A. (2008), « Les services à la personne en 2006 : une croissance continue ", Premières synthèses, Dares, $\mathrm{n}^{\circ} 48.2$, novembre.

Damesin R., Pelisse J. (2004), « Le droit du travail : une ressource inégale dans les entreprise ", in $L a$ lettre $d u$ GIP, $\mathrm{n}^{\circ} 20$. 
Duriez B., Nizey J. (2002), « Les aides familiales. $\mathrm{Du}$ mouvement à l'association de services », in Chauviere M., Duriez B., Nizey J., La solidarité en actes. Services collectifs et expression des usagers dans le mouvement populaire des familles, 19401955, Paris, Les Cahiers du GRMF, n 11.

Dussuet A. (2005), Travaux de femmes : enquête sur les services à domicile, Paris, L'Harmattan.

Dussuet A. (2009), « Les dispositifs de prévention des risques de santé dans les associations d'aide à domicile », Contribution au deuxième séminaire du réseau Care : système d'emploi et professionnalisation, Paris, Cnam, 28 mai.

Dussuet A., Loiseau D. (2007), « Les services aux familles offertes par les associations : un modèle de service « entre » formel et informel ? », in Dussuet A., Lauzanas J.-M., L'économie sociale entre informel et formel: paradoxes et innovations, Presses Universitaires de Rennes, Rennes.

Hely M. (2009), Les métamorphoses du monde associatif, Paris, Presses Universitaires de France.

Hughes E. C. (1952), American Journal of Sociology, volume $57, \mathrm{n}^{\circ} 5$, mars.

Jany-Catrice F. (2008), « Services à la personne : le choix de la France en faveur de l'inégalité » in Maurin L. Savidan P. L'état des inégalités en France, Paris, Éditions Belin.

Jany-Catrice F. (2009), « L'invention politique d'un secteur : le cas des services à la personne ", Paris, Séminaire OFCE-IRES, 18 mars.

Jany-Catrice F., Puissant E. (2010), « L'aide à domicile face aux services à la personne et registres d'action contradictoires : des politiques aux organisations ", in Revue de l'Ires, n 64.

Lefebvre M. (2010), « Qualité de l'emploi et hétérogénéité dans le champ des services à la personne : éléments d'analyse à partir des statistiques natio- nales », Documents de travail du Clersé (Working Papers), ${ }^{\circ} 4$, février.

Leplay B. (2005), « Gestion des conflits dans les petites entreprises », in Denis J.-M., Le conflit en grève? Tendances et perspectives de la conflictualité contemporaine, Paris, La Dispute.

Puissant E. (2006), Les conflits du travail dans les associations d'aide à domicile aux personnes âgées en voie de professionnalisation, mémoire de master réalisé sous la co-direction de Danièle Demoustier et d'Olivier Mériaux, IEP de Grenoble, soutenu en septembre.

Puissant E. (2007), « Les associations d'aide à domicile : des tensions dans la relation de travail », Contribution aux Journées de l'AES, Nanterre, Septembre.

Puissant E. (2010), La relation associative d'aide à domicile; spécificités, remises en cause, résistances, Grenoble, thèse de doctorat, soutenue publiquement le 7 décembre.

Reynaud J.-D. (1995), Le conflit, la négociation et la règle, Toulouse, Octarès.

Salais R., Storper M. (1993), Les mondes de production : enquête sur l'identité économique de la France, Paris, Éditions de l'EHESS.

Vasselin K. (2002), « Faire le ménage : de la condition domestique à la revendication d'une professionnalité », in Piotet F., La révolution des métiers, Paris, Presses Universitaires de France.

Vienney C. (1994), L'économie sociale, Paris, La Découverte. 


\title{
Résumé
}

\section{Le rôle ambivalent des associations d'aide à domicile dans la professionnalisation des emplois et des salariées}

\author{
Emmanuelle Puissant
}

Les associations d'aide à domicile ont engagé, depuis les années 70, un large processus de professionnalisation. Quarante ans après cette amorce, elles jovent un rôle ambivalent quant à la professionnalisation des emplois et des salariées du secteur. En effet, elles participent au maintien des employées dans un rapport domestique à leur travail, mais en tant qu'acteur collectif, elles réunissent aussi les conditions pour re-collectiviser un travail isolé et éclaté. Or, la création d'un espace collectif de travail, dans ce secteur nous paraît être une condition à la reconnaissance de ces activités comme constituant un véritable métier, requérant des compétences professionnelles particulières qui s'acquièrent.

Mots clés

Service aux particuliers, aide à domicile, professionnalisation, savoir professionnel Journal of Economic Literature : L 84, L 31 